\title{
Im Doppelpack hoch effektiv
}

\author{
Senkung von Blutdruck und LDL verlängert das Leben deutlich
}

Sowohl das Risiko als auch der Nutzen gehen bei Blutdruck und LDL-Cholesterin Hand in Hand. Während erhöhte Werte das Risiko für kardiovaskuläre Erkrankungen drastisch erhöhen, kann eine gleichzeitige Senkung beider Parameter das Leben deutlich verlängern, wie Dr. Brian Ference aus Detroit beim europäischen Kardiologenkongress in Rom berichtete.

Ließen sich Blutdruck- und LDL-Cholesterinwerte gleichzeitig auch nur moderat über eine sehr lange Zeit verringern, könnten Herzinfarkte und Schlaganfälle so gut wie eliminiert werden. Zu diesem Ergebnis kamen Ference und seine Mitarbeiter in einer Analyse einer jahrzehntelangen Beobachtungsstudie mit eingebauter „natürlicher Randomisierung“, die auch Schlüsse aus Daten nichtrandomisierter Beobachtungsstudien hinsichtlich kausaler Zusammenhänge zulässt. Dazu werden genetische Polymorphismen genützt, die in Zusammenhang damit stehen, ob beispielsweise Blutdruck- oder Cholesterinwerte einer Person lebenslang höher oder niedriger sind. Die natürliche Randomisierung solcher Polymorphismen wird dabei als Zufallsfaktor herangezogen. Ein bestimmtes Allel zu erben, das spezifisch mit niedrigeren LDL-Cholesterinwerten assoziiert ist, wäre demnach quasi analog zu einer Randomisierung auf eine LDL-senkende Therapie in einer Langzeitstudie.

Um zu klären, welchen kausalen Effekt niedrigere im Vergleich zu höheren Blut- druck- und LDL-Werten auf das Risiko für kardiovaskuläre Ereignisse haben, wurden genetische und kardiovaskuläre Daten von 102.000 Personen herangezogen, die an 14 Kohorten- oder Fall/Kontroll-Studien teilgenommen hatten und über einen Zeitraum von bis zu 32 Jahren nachbeobachtet worden waren. Auf Basis erblicher Polymorphismen, die mit Blutdruck und LDLCholesterin assoziiert sind, wurde für jede Person ein genetischer Score gebildet.

\section{Unabhängig, multiplikativ und kumulativ}

Anhand dieser Scores wurde dann eine Einteilung in vier Gruppen vorgenommen, bestehend aus einer Referenzgruppe, einer Gruppe mit niedrigeren LDL-Spiegeln, einer Gruppe mit niedrigerem systolischen Blutdruck und einer Gruppe mit niedrigeren Werten für beide Parameter. Im Verlauf von mehr als drei Jahrzehnten wurden 14.368 schwerwiegende vaskuläre Erstereignisse erfasst, darunter koronar bedingte Todesfälle, Myokardinfarkte, Schlaganfälle und koronare Revaskularisationen. Schon bei Personen, die im Vergleich zur Referenzgruppe niedrigere LDL- oder Blutdruckwerte hatten, war das relative Risiko für diese kardiovaskulären Ereignisse jeweils deutlich niedriger (um 54,2 Prozent respektive 44,7 Prozent). In der Gruppe, in der sowohl die LDL- als auch Blutdruckwerte niedriger waren, war das Ergebnis noch beeindruckender: Langfristig um 1 mmol/l (39 mg/

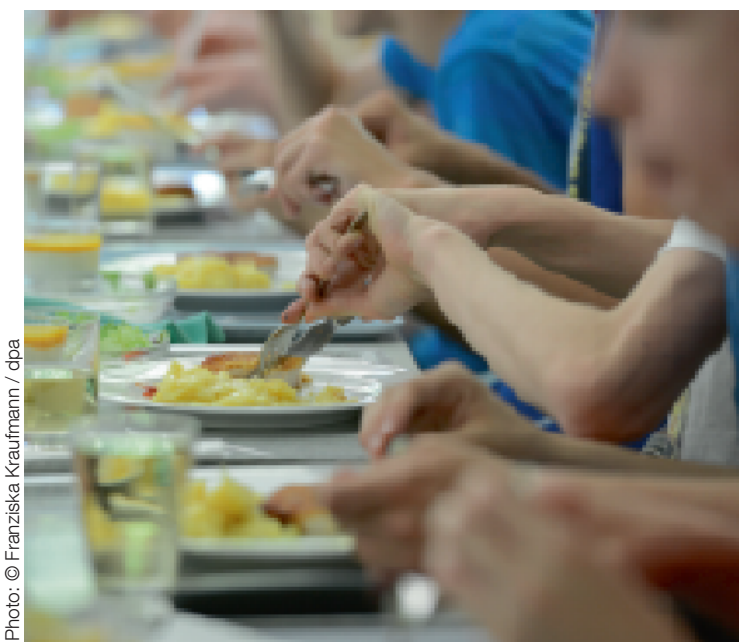

Prävention beginnt beim Essen und Im Kindesalter.

dl) niedrigere LDL-Werte und zugleich um $10 \mathrm{mmHg}$ niedrigere systolische Blutdruckwerte gingen mit einem um 86,1 Prozent niedrigeren Risiko einher.

„LDL-Cholesterin und systolischer Blutdruck haben unabhängige, multiplikative und kumulative kausale Effekte auf das Risiko für kardiovaskuläre Ereignisse“, so Ference. Deshalb habe die Kombination aus langfristig niedrigeren LDL- und Blutdruckwerten das Potenzial, das Lebenszeit-Risiko für entsprechende Ereignisse dramatisch zu senken. Dazu seien Präventionsprogramme nötig, um LDLCholesterin und Blutdruck möglichst frühzeitig im Leben zu senken.

$\mathrm{red} / \ddot{A} Z / p o$

\section{IMPRESSUM}

Herausgeber und Verleger: Springer-Verlag GmbH, Prinz-Eugen-Str. 8-10, Postfach 11, 1040 Wien, Austria, Tel.: $01 / 33024$ 15, Fax: $01 / 3302426$ Internet: www.springer.com, www.SpringerMedizin.at Geschäftsführung: Joachim Krieger, Dr. Alois Sillaber, Dr. Heinrich Weinheimer Leitung Journale und Redaktionen Medizin: Gabriele Hollinek Redaktion: Verantwortlicher Redakteur: Verena Kienast, Dipl. Tzt. Elise Haidenthaller Leitung Verkauf Medizin: Robert Seiwald Anzeigen: Gabriele Popernitsch Mediaservice: Claudia Trischler; Es gilt an Anzis zug A dingung zugsed

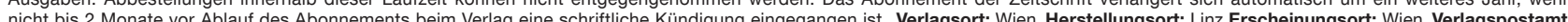
1040 Wien P. b. ISSN Print: 0949-7323, ISSN Eloctronic: 1613-7574, Band 21, Heft 9/2016 Layout: KM-Satz Design: Wojtek Grzymala Druck: Friedrich Druck \& Medien GmbH L 1040 Wien P. b. b. ISS Print. 0949-7323, ISSN Electronic. 1613-7574, Band 21, Heft 9/2016 Layout: KM-Satz Design: Wojtek Grzymala Druck: Friedrich Druck \& Medien GmbH, Linz, Aust-

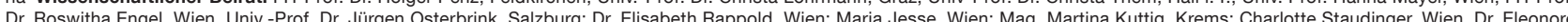
Dr. Roswïha Enge, Wen, Univ.-Prof. Dr. Jürgen Osterbrink, Salzburg, Dr. Elsabeth Rappold, Wien; Maria Jesse, Wien; Mag. Martina Kuttig, Krems; Charlotte Staudinger, Wien. Dr. Eleonore bereich des Verfas. A Rela Einschaltungen nach \$26 Mediengesetz. Allgemeiner Teil/Rechtliche Hinweise für Autoren Die Autorin/der Autor erklärt, dass ihr/sein Manuskript in dieser Form bislang nicht anderweitig veröffentlicht oder zur Veröffentlichung eingereicht wurde. Die Autorin/der Autor überträgt mit der Übergabe des fertigen Manuskripts und der Veröffentlichung in der Fachzeitschrift die notwendigen fentlicht oder zur VerVerielältigung und Verbreitung an Autorin/der Autor ubertagl Nutzungsrechte zur Vervielfaltigung und Verbreitung an den Verlag, insbesondere das Recht der Nüzung zu gewerblichen Zwecken durch Druck, Nachdruck, Verbreitung in elektronischer Form oder andere Verfahren und Medien durch Springer Nature. Beitrage, die in procare erscheinen, Können auch in der Springer-Zeitschrift Heliberufe veroffentlicht werden. Die Autorin/der Autor holt, falls notwendig, die Nutzungsrechte an Texten und Bildern Dritter vor Ubergabe des fertigen Manuskripts ein, eventuelle Ansprüche Dritter sind somit geklärt. Hinweise zur Verwertung:
Die Zeitschrift sowie alle in ihr enthaltenen einzelnen Beiträge und Abbildungen sind urheberrechtlich geschützt. Jede Verwertung, auch auszugsweise, die nicht ausdrücklich vom Urheber-

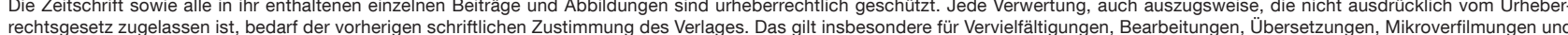
rechtsgesetz zugelassen ist, bedarf der vorherigen schriftlichen Zustimmung des Verlages. Das gilt insbesondere für Vervielfältigungen, Bearbeitungen, Übersetzungen, Mikroverfilmungen und
die Verarbeitung in elektronischen Systemen. Produkthaftung: Die Wiedergabe von Gebrauchsnamen, Handelsnamen, Warenbezeichnungen usw. in dieser Zeitschrift berechtigt auch ohne die Verarbeitung in elektronischen Systemen. Produkthaftung: Die Wiedergabe von Gebrauchsnamen, Handelsnamen, Warenbezeichnungen usw. in dieser Zeitschrift berechtigt auch ohne besondere Kennzeichnung nicht zu der Annahme, dass solche Namen im Sinne der Warenzeichen- und Markenschutz-Gesetzgebung als frei zu betrachten waren und daher von jedermann Verlag übrnimmt hirfür keine Gewähr Eigentümer und Copyright-Inhaber: ( ) 2016 Springer-Verlag/Wien. Springer Medizin ist Teil von Springer Nature Coverbild: O Sherry Young / Fotolia 